\title{
Performance Evaluation of Rotational Interleaver for IDMA Scheme in Acoustic Environment
}

\author{
Prachi Tripathi, Shivani Dixit, M. Shukla \\ Harcourt Butler Technological Institute, Kanpur, India \\ Email: manojkrshukla@yahoo.in
}

Received 5 May 2014; revised 18 June 2014; accepted 28 July 2014

Copyright (C) 2014 by authors and OALib.

This work is licensed under the Creative Commons Attribution International License (CC BY). http://creativecommons.org/licenses/by/4.0/

(c) (i) Open Access

\begin{abstract}
The whole globe is covered universally by water with a majority space. In view of extraction of minerals as well as national security reasons, underwater communication has become quite important for all the mankind. Researchers, around the world are working for optimizing the multiple scheme in acoustic channel. Recently, the researchers have reported valuable employability of IDMA scheme in acoustic communication. In IDMA Scheme, the users are distinguished with userspecific interleaving sequences based on chip-level interleaving mechanism instead of user-specific signature sequences as engaged in CDMA scheme. However the role regarding formation of interleavers plays vital notch for performance of IDMA scheme in underwater communication. In this paper, the performance of user-specific rotational interleaver has been observed in acoustic environment for BPSK signaling scheme on MATLAB platform. Simulation results demonstrate that the rotational interleaver performs better in terms of bit error rate (BER) for underwater channel employing iterative IDMA system.
\end{abstract}

\section{Keywords}

Iterative IDMA System, Underwater Communication, BER, Rotational Interleaver

Subject Areas: Mobile and Portable Communications Systems, Mobile and Ubiquitous Networks, Simulation/Analytical Evaluation of Communication Systems

\section{Introduction}

The interleave division multiple access (IDMA) scheme has emerged as most promising schemes in horizon of acoustic communication. This scheme defines the idea of interleaving as the only means for user separation. The IDMA system [1] [2] has been reported with better bit error rate (BER) in comparison to conventional CDMA 
systems with superior robustness against fading and cross-cell-interference. It also concedes a very simple chipby-chip multiuser detection (CBC-MUD) layout while accomplishing impressive BER performance. For multipath channel with IDMA scheme, the per user complexity of multiuser detection (MUD) algorithm, is quite less [2]. Therefore, for higher count of users, CBC-MUD mechanism presents better results [3] [4] in any communication environment.

In literature, BER performance of IDMA system has been presented employing randomly and autonomous generating interleavers. The simulation results demonstrate better BER performance correspondingly and even better than an analogous CDMA system [5].

The rest of the paper is organized as: Section 2 is devoted to introduction to acoustic underwater communication. The concept of IDMA scheme has been presented in Section 3 while Section 4 illustrates the mechanism of interleavers and importance of orthogonality in IDMA scheme. The mechanism of rotational interleaver has been provided in Section 5 while simulation results and conclusions have been presented in Section 6 and Section 7 respectively.

\section{Underwater Acoustic Communication}

Underwater sensor networks have some similarities with their ground-based counterparts such as their structure, function, computation and energy limitations. It is well known fact that the radio waves do not propagate well underwater due to the high energy absorption created by underwater channel. The creatures inside sea-level use acoustic waves for propose of normal communication. Therefore, underwater communication systems are designed to be based on acoustic links characterized by large population delays. The propagation speed of acoustic signals, in water (typically $1500 \mathrm{~m} / \mathrm{s}$ ) is five orders of magnitude lower than the radio wave propagation speed in free space [6].

Acoustic channels are known for its limitation for accommodating low bandwidth for signal. Therefore link quality in underwater communication is severely affected by multipath, fading, and the refractive properties of the sound channel resulting in high bit error rates for acoustic links with losses of connectivity as predicted in Figure 1. The signals transmitted from source are not only facing scattering effect but also are being reflected with upper sea level as well surface of the earth. In addition to it, there are various unevenly spread time varying sound-speed profiles in whole the sea. These sound-speed profiles also affect the user data adversely.

Although, in underwater applications, certain communication nodes are anchored to the bottom of the ocean, however, various other applications require sensors to be suspended at certain depths or to move freely in underwater medium. Therefore, underwater sensors, installed below and upon sea-surface, change their physical locations with water currents. It finally portraits the future development of geographical routing to be very promising in underwater communication and networking due to its scalability and limited signaling properties. However, it cannot rely on the global positioning system (GPS) because it uses radar waves in the $1.5 \mathrm{GHz}$ band that do not propagate in water [6] [7].

Underwater communication systems have more stringent power requirements than terrestrial system because acoustic communications are more power hungry, and typical transmission distances are greater, hence higher power is required to ensure coverage. In addition to it, it is really extremely difficult to change power banks for communications systems under the sea level. Therefore, an energy efficient design of underwater communication system is quite mandatory.

For incorporating the data related to various users within certain bandwidth and limited power profile, suitable multiple access scheme has to employed. Earlier, the researchers have suggested the application of various multiple access schemes including CDMA scheme and OFDMA scheme however they are built in with certain merits and demerits. Recently, the researcher communality has reported the claim of IDMA scheme in underwater communication, which incorporates various merits of CDMA and OFDMA schemes while relaxing some of major demerits of aforesaid schemes including mitigation against fading and multiple access interference [5].

\section{Iterative IDMA Scheme}

An iterative IDMA system [1] shown in Figure 2, there are k simultaneous user with single path acoustic channel. In transmitter end, $N$-length input data sequence $d_{k}=\left[d_{k}(1), d_{k}(2), \cdots, d_{k}(N)\right]$ of user $k$ is encoded into chips $c_{k}=\left[c_{k}(1), c_{k}(2), \cdots, c_{k}(j)\right]$ based on low rate code $c$ or foreword error control FEC, where $j$ is the chip length. The term $c_{k}$ is permutation by an interleaver $\pi_{k}$ for user-specific data. 


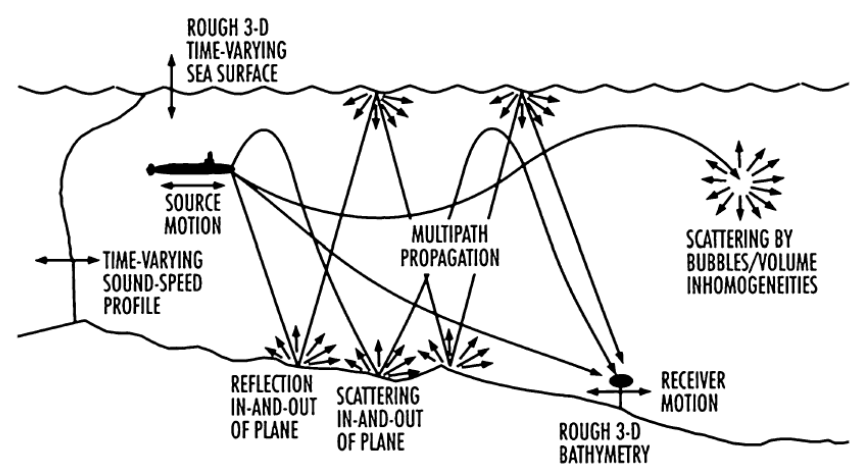

Figure 1. Scenario of acoustic communication.

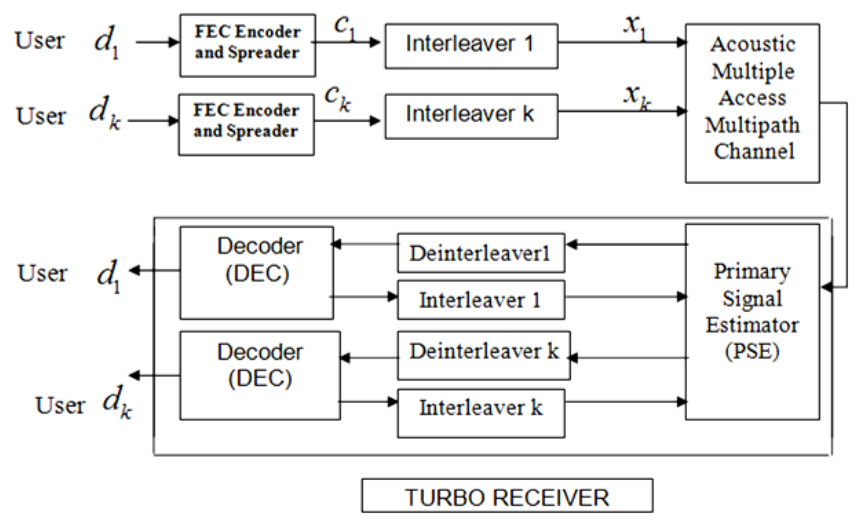

Figure 2. Acoustic IDMA system.

$$
x_{k}=\left[x_{k}(1), x_{k}(2), \cdots, x_{k}(j)\right]
$$

Elements in $x_{k}$ are called “chips” for IDMA conventional users who are solely distinguished by their interleavers [6].

Hence the name interleave division multiple access (IDMA) defines interleavers $\pi_{k}$ for various users. These interleavers are generated independently and randomly. Basically interleavers are rearranged sequences of strings of user-specific data. It is in form of coded sequences, so that adjacent chips are approximately uncorrelated. In forward error correction coder (FEC) block, suitable channel coding is introduced which is further decoded accordingly in decoder (DEC) block in the receiver section. In turbo receiver block, the user-specific data is retrieved after suitable count of iterations.

\subsection{The ESE Function}

The Output of the elementary signal estimator (ESE) and decoders (DECs) are extrinsic log-likelyhood ratio (LLRs) for user data $\left\{x_{k}(j)\right\}$ define below. The estimation of user data for specific $j^{\text {th }}$ chip is given by,

$$
e\left(x_{k}(j)\right)=\log \left(\frac{p\left(y / x_{k}(j)=+1\right)}{p\left(y / x_{k}(j)=-1\right)}\right), \forall k, j
$$

The signal estimates $e_{\mathrm{ESE}}\left(x_{k}(j)\right)$ and $e_{\mathrm{DEC}}\left(x_{k}(j)\right)$, depends upon whether they are generated by the ESE or DECs blocks.

Assume that channel has no memory after chip-match filtering; the received signal from $k$-users can be written as

$$
r(j)=\sum_{k=L}^{K} h_{k} x_{k}(j)+n(j)
$$

where $j=1,2, \cdots, J$ 
$h_{k}=$ channel co-efficient for user- $k$ and $n(j)$ are samples of an AWGN process with variance $\sigma^{2}=N_{0} / 2$. $r(j)$ is one sample used one at a time.

$$
\begin{gathered}
r(j)=h_{k} x_{k}(j)+\varepsilon_{k}(j) \\
\varepsilon_{k}(j)=r(j)-h_{k} x_{k}(j) \\
\varepsilon_{k}(j)=\sum_{k^{\prime}=k}\left(h_{k^{\prime}} x_{k^{\prime}}(j)+n(j)\right)
\end{gathered}
$$

Equation (4) shows distortion in user-specific received signal with respect to all the other users including channel noise [7].

From the central limit theorem, $\varepsilon_{k}(j)$ can be approximated as a Gaussian variable and $r(j)$ can be characterized by a conditional Gaussian probability density function

$$
P\left(\left(r_{j} \mid x_{k}(j)\right)= \pm 1\right)=\frac{1}{\sqrt{2 \pi} \operatorname{var}\left(\varepsilon_{k}(j)\right)} \exp \left(\frac{r(j)-\left( \pm h_{k}+E\left(\varepsilon_{k}(j)\right)^{2}\right)}{2 \operatorname{var} \varepsilon_{k}(j)}\right)
$$

Further, central limit theorem is applied to the summation of a larger no of random variables.

$$
\begin{gathered}
e_{\mathrm{ESE}}\left(x_{k}(j)\right)=\frac{1}{\sqrt{2 \pi \operatorname{var}\left(\varepsilon_{x}(j)\right)}} \log \left[\exp -\left[\frac{\left(r(j)-E\left(\varepsilon_{k}(j)-h_{k}\right)\right)^{2}}{2 \operatorname{var} \varepsilon_{k}(j)}-\frac{\left(r(j)-E\left(\varepsilon_{k}(j)+h_{k}\right)\right)^{2}}{2 \operatorname{var} \varepsilon_{k}(j)}\right]\right] \\
e_{\mathrm{ESE}}\left(x_{k}(j)\right)=2 h_{k} \cdot \frac{r(j)-E\left(\varepsilon_{k}(j)\right)}{\operatorname{var} \varepsilon_{k}(j)}
\end{gathered}
$$

\subsection{The DEC Function}

With BPSK signaling, their output is the extrinsic LLRs $\left\{e_{\mathrm{DEC}}\left(x_{k}(j)\right)\right\}$ of $\left\{x_{k}(j)\right\}$ is defined in (1) which are used to generate the following statistics. The user-specific estimate and variance can be calculated by,

$$
\begin{gathered}
E\left(x_{k}(j)\right)=\tanh \left(e_{\mathrm{DEC}}\left(x_{k}(j)\right)\right) / 2, \\
\operatorname{var}\left(x_{k}(j)\right)=1-\left(E\left(x_{k}(j)\right)\right)^{2}
\end{gathered}
$$

The log-likelyhood ratio (LLR) output of DEC function is given by,

$$
e_{\mathrm{DEC}}\left(x_{k}\left(\pi_{j}\right)\right)=\sum_{j=1}^{s} \log \left(\frac{p\left(r\left(\pi_{k}(j)\right) \mid x_{k}\left(\pi_{k}(j)\right)=+1\right)}{p\left(r\left(\pi_{k}(j)\right) \mid x_{k}\left(\pi_{k}(j)\right)=-1\right)}\right)=\sum_{j=1}^{s} e_{\mathrm{ESE}}\left(x_{k}\left(\pi_{k}(j)\right)\right)
$$

In fact, decoder (DEC) block is responsible for retrieving the user-specific data after channel decoding process.

\section{Interleaving Mechenism}

In CDMA scheme, interleaving is basically used as the most popular combination to correct burst errors only. However, in IDMA scheme, for generation of user-specific interleavers, suitable for different users, orthogonal interleaving mechanism is taken into use.

The term "interleaving" is defined as permutation rule that scrambles data to separate distinct locations. In the field of communication engineering, data is initially interleaved on the transmitter while it is duly de-interleaved on received side with the help of user-specific interleavers. Appropriate orthogonal interleavers, suitable for IDMA scheme, must inherit effects such as easy implementation with lower complexity and minimum number of collisions among them. Minimum collisions amongst user-specific interleavers, is termed to be an important criterion because they are used for user separation in IDMA scheme.

The term "orthogonality" is also an important factor for generating the interleavers for IDMA scheme. The 
perfectly orthogonal interleavers have zero cross correlation amongst each other in order to minimize the multiple access interference (MAI) between the data related to various users. The correlation between them increases with increment in user count and consequently BER degrades. The orthogonality criteria clearly indicated regarding minimum count of collisions amongst each other [4].

The researchers have reported various mechanism for generation of orthogonal interleavers including random interleaver [1]-[5], rotational interleaver [7], and prime interleaver [8] [9] however some interleavers are observed to be better in terms memory requirement while others are better in terms of generation complexity and BER performance amongst various other quality parameters.

In Figure 3, the memory requirement of all three interleaver has been demonstrated for count of 8 users. The simulation results predict the suitability of rotational interleaver for scheme.

In the next section, the simulation results are presented for fixed count of iterations, data length and spread length in transmitter and receiver sections. The simulations have been performed with MATLAB environment.

\section{Simulation Results}

For simulation purpose, single cell environment has been assumed with IDMA scheme. Further various interleavers have also been simulated on such scheme with one transmitter and one receiver architecture. The signaling scheme is opted to be BPSK. Also the entire users are assumed to bear same data length. In general, 10 iterations have been performed for extracting the user-specific data in receiver section while block length is opted to be 200 bits for simulation purpose.

In Figure 4, the performance of IDMA scheme has been observed in underwater acoustic (UWA) channel and AWGN channel for 16 users. The simulation results demonstrate the inferior BER performance in case of UWA channel due complexity and several disturbances involved in communication under the surface of water.

During evaluation of performance for IDMA scheme is observed for 8 users and 16 users with rotational interleaver in UWA channel in Figure 5, the performance of the system dips for higher user count. It happens due to increment in MAI in case of increment in user count.

Figure 6 presents the performance of IDMA scheme for random, prime and rotational interleaving mechanisms in UWA channel with variation in data length. The simulation results demonstrate the satisfactory performance of rotational interleavers for both data length of 256 and 512 bits. The performance of the system improves for higher data length due increment in availability of higher count of interleavers with hike in data length. In this case, for multiple users, data length is opted to be 1024 bits with 16 spreading factors.

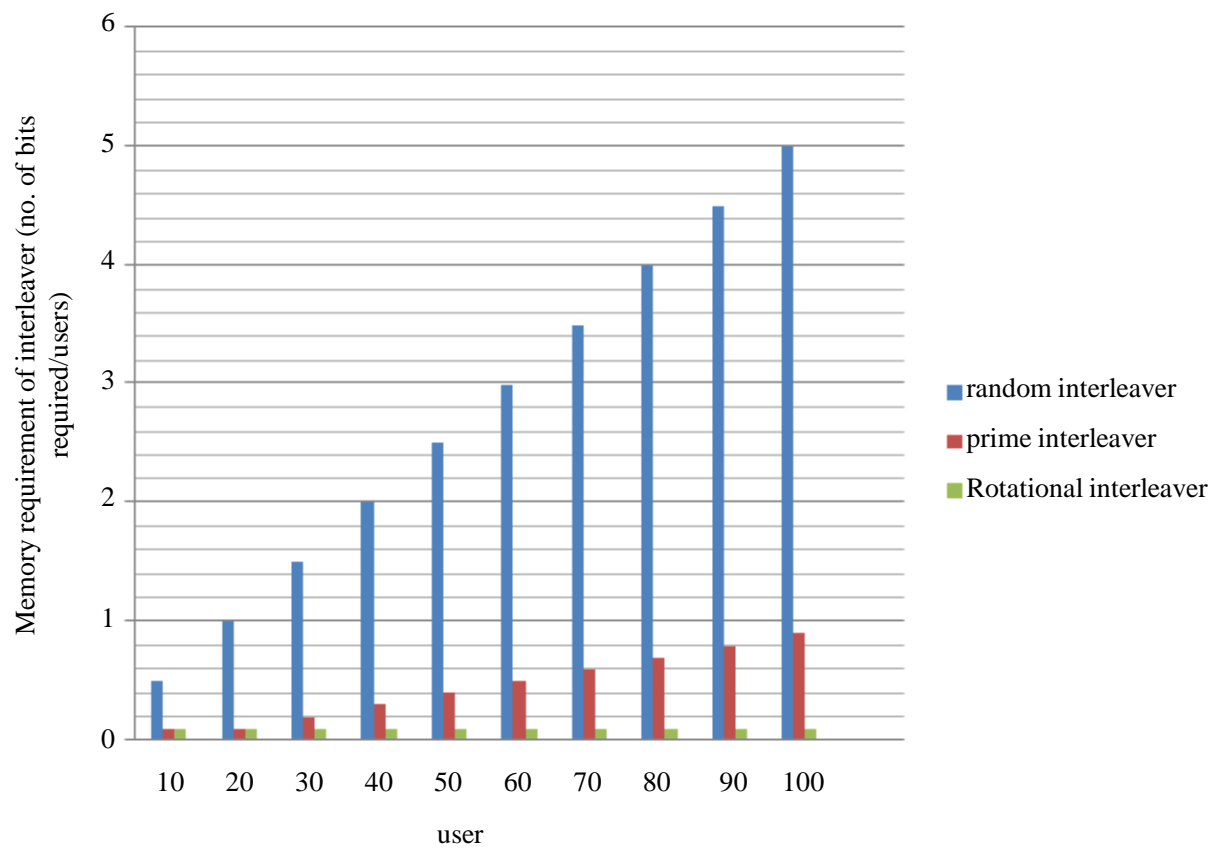

Figure 3. Memory requirement of various interleavers. 


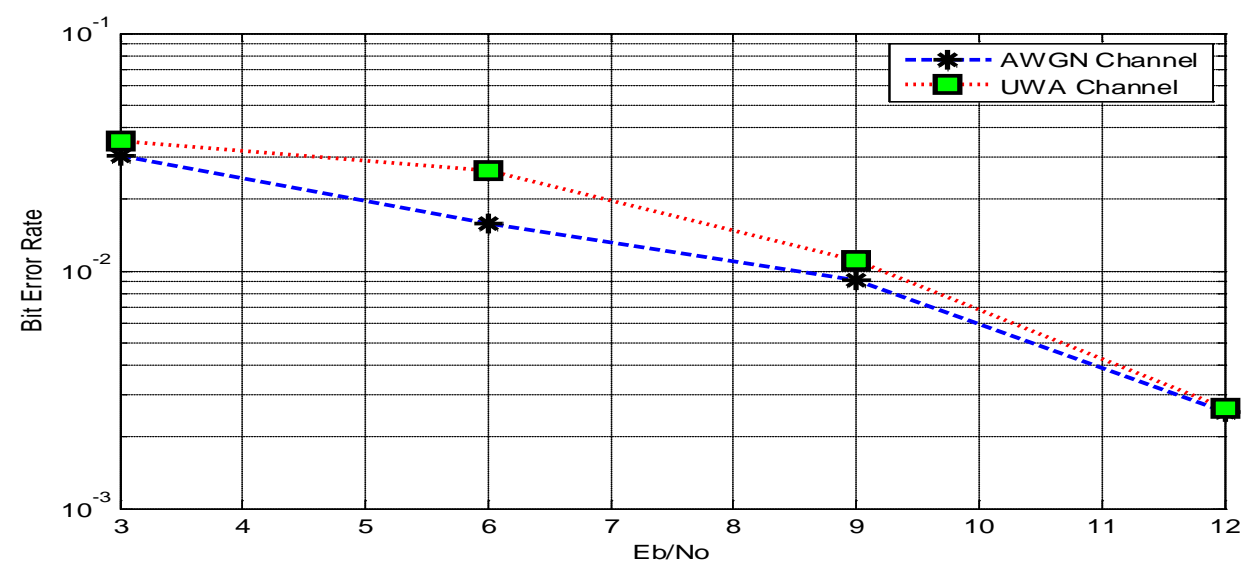

Figure 4. Rotational interleaver in UWA and AWGN channel.

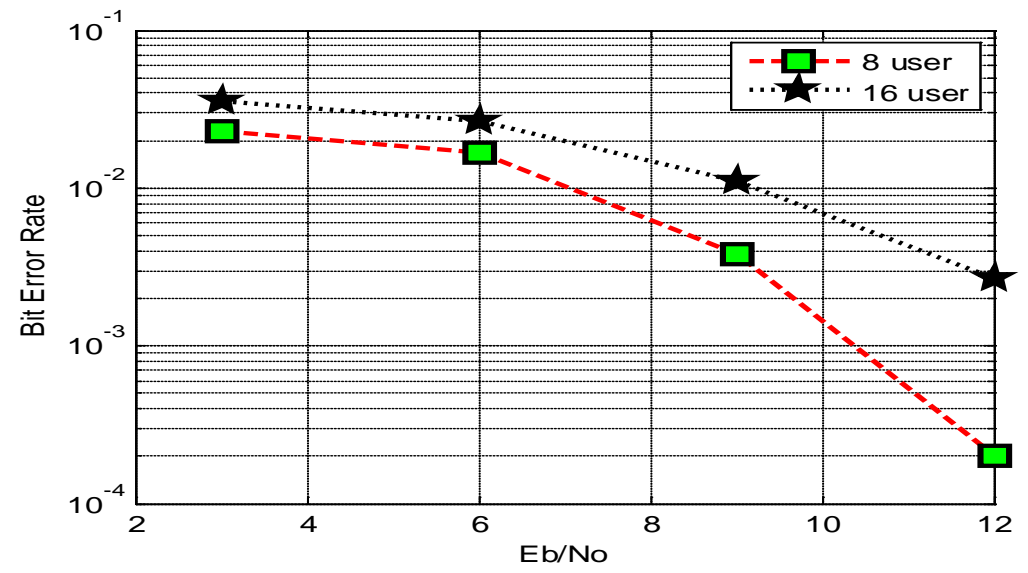

Figure 5. Rotational interleaver for various users.

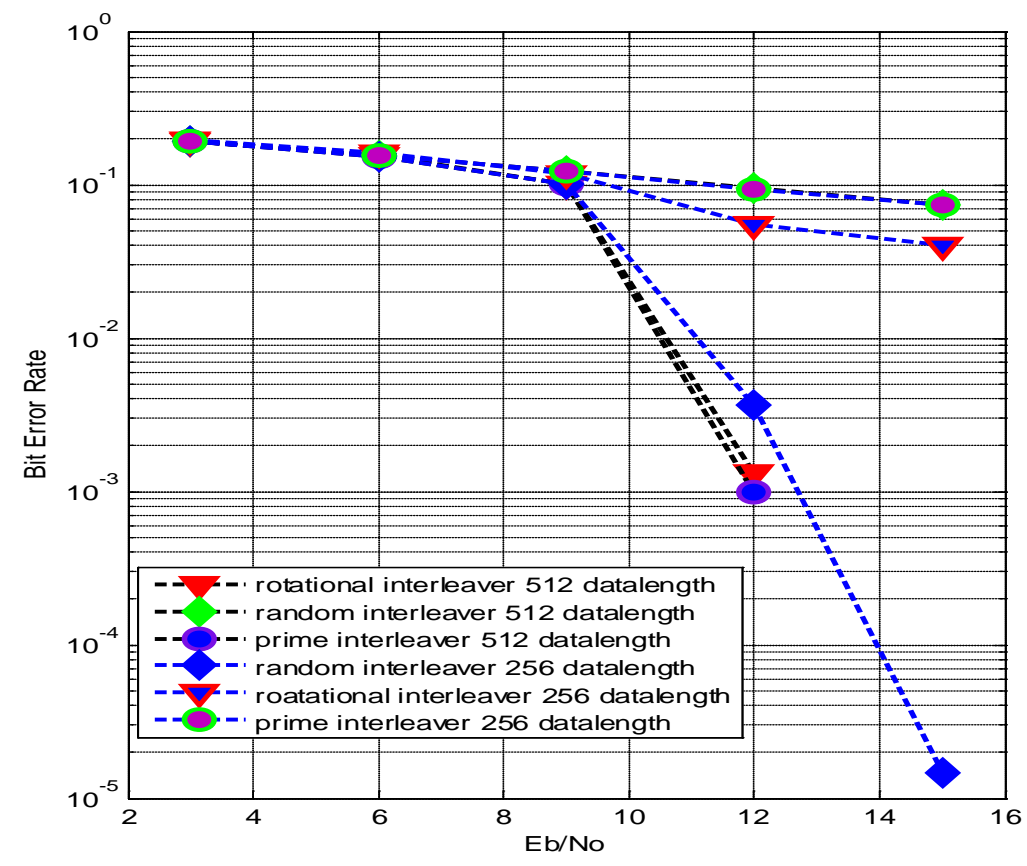

Figure 6. Peformance of interleavers for variation in data length. 
Simulation results demonstrate that rotational interleaver perform well than in presented scheme for simulations in term of lower BER for same number of users.

\section{Conclusions}

After observing the simulation results, it can be concluded that the rotational interleaver performs similar to other interleaving mechanism in terms of BER however outperforms other interleaving mechanism in terms of memory requirement at transmitter and receiver ends for generation and storing of user-specific interleavers.

The IDMA scheme performs satisfactorily in UWA communication environment with various interleavers. The results are simulated with underwater with IDMA in MATLAB environment. From the simulation results, it is clear that IDMA scheme could be used with UWA channel also with good BER performance. However, for combating multipath fading, suitable diversity mechanism can be adopted along with proper RAKE receiver.

\section{Funding}

The works has been supported by TEQIP II Project running under World Bank through U.P. State Government, India.

\section{References}

[1] Li, P. and Liu, L. (2006) Interleave Division Multiple-Access. IEEE Transaction on Wireless Communication, 5, 938-947.

[2] Wang, X. and Poor, V. (1999) Iterative (Turbo) Soft Interference Cancellation and Decoding of Coded CDMA. IEEE Transaction on Communication, 47, 1046-1061. http://dx.doi.org/10.1109/26.774855

[3] Shukla, M., Srivastava, V.K. and Tiwari, S. (2006) Iterative Division Multiple Access: New Trend in Wireless Communication. Proceedings NCCT Conference, India, 151-154.

[4] Shukla, M., Srivastava, V.K. and Tiwari, S. (2009) Analysis and Design of Optimum Interleaver for Iterative Receiver in IDMA Scheme. Wiley Journal of Wireless Communication and Mobile Computing, 9, 1312-1317.

[5] Pande, T., Gupta, K., Shukla, M., Tripathi, P. and Singh, A. (2014) Underwater Communication with IDMA Scheme. Intelligent Computing, Networking, and Informatics, Proceedings of ICACNI Conference, India, 243, 1171-1177.

[6] Stojanovic, M. and Preising, J.C. (2009) Underwater Acoustic Communication Channels: Propagation Models and Statistical Characterization. IEEE Communications Magazine Homepage, 47, 84-89. http://dx.doi.org/10.1109/MCOM.2009.4752682

[7] Gupta, K., Shukla, C.K., Sharma, S., Saxena, A. and Shukla, M. (2012) Rotational Interleaver for Iterative-Division Multiple-Access Scheme. 2012 International Conference on Communication Systems and Network Technologies (CSNT), Rajkot, 11-13 May 2012, 635-638.

[8] Shukla, M. and Gupta, R. (2010) Performance Analysis of Optimum Interleaver based on Prime Numbers for Multiuser Iterative IDMA Systems. International Journal of Interdisciplinary Telecommunications and Networking (IJITN), 2, 51-65. http://dx.doi.org/10.4018/jitn.2010070103

[9] Shukla, M., Akanksha, G. and Bhatia, R. (2011) Performance Evaluation of Maximal Ratio Receiver Combining Diversity with Prime Interleaver for Iterative IDMA Receiver. International Journal of Information Engineering and Applications, 1, 29-39. 